Abb. 1 Gastrointestinale Erkrankungen wie das Reizdarmsyndrom betreffen $20-30 \%$ aller Menschen. Quelle: Kirsten Oborny, Thieme Gruppe

\title{
Verdauung neu lernen
}

\section{Das ENTERISCHE NERVENSYSTEM und seine Regulationsstörungen am Beispiel Reizdarmsyndrom. Keren Grafen}

\section{KURZ GEFASST}

1 Das enterische Nervensystem (ENS) steuert die Motilität, Sekretion, Versorgung und Abwehr des Darmes und ist mit 150 Millionen Neuronen die größte Ansammlung von Nervenzellen außerhalb des Zentralnervensystems.

2 Zu den ENS-assoziierten gastrointestinalen Störungen zählen Morbus Hirschsprung, Achalasie, die Chagas-Krankheit und als Leiterkrankung das Reizdarmsyndrom.

3 Am Beispiel des Reizdarmsyndroms lässt sich die effektive Therapie von ENS-Störungen in Form einer Kombination aus Ernährungsumstellung, Stressreduktion und orthomolekularer Versorgung zeigen.
In den letzten zehn Jahren wurden mehr als 20 ursächliche Faktoren funktionaler gastrointestinaler Erkrankungen identifiziert. Doch wie lassen sich diese kausal behandeln? Vielfach ist das enterische Nervensystem pathophysiologisch beteiligt, mit dem Reizdarmsyndrom als Leiterkrankung. Physiologisch manifestiert sich die Krankheit, unabhängig von den Ursachen, immer in einer gestörten nervösen Regulation der glatten Muskulatur und/oder der Schleimhaut. Dies führt letztendlich zu einem Nervenzellverlust im Darm. Man spricht von enterischer Neuropathie.

Doch welche funktionelle und anatomische Struktur liegt dem enterischen Nervensystems (ENS) zugrunde, wie interagiert es mit dem Nerven- und Immunsystem des Körpers, und welche Erkrankungen und Therapieansätze ergeben sich aus seinen Störungen? 


\section{Zentrum für Funktion, Versorgung und Immunsystem des Darms}

Das enterische Nervensystem koordiniert als eigenständige Steuerung lokale Vorgänge im Darm, insbesondere die Motorik, die für die Motilität (zum Beispiel den Transport der Speisen durch den Verdauungstrakt) notwendig ist. Mit eingeschlossen ist die Sekretion der dazugehörigen Drüsen (Speicheldrüse, Pankreas, Gallenblase) und die bedarfsadaptierte Durchblutung des Verdauungstrakts. Im Rahmen neuroimmunologischer Vorgänge ist das enterische Nervensystem auch an der Abwehr von Krankheitserregern und an Entzündungsprozessen beteiligt.

Merke: Der englische Physiologe John Langley prägte 1921 den Begriff des enterischen Nervensystems. Mit seinen 150 Millionen Neuronen handelt es sich dabei um die größte Ansammlung von Nervenzellen außerhalb des Zentralnervensystems.

\section{Zusammenwirken von Nerven- und Gliazellen}

Die kleinste Funktionseinheit des enterischen Nervensystems bilden Neuronen und Gliazellen. Letztere sind ähnlich wie im Gehirn für die Signalverarbeitung beziehungsweise Versorgung der Neuronen zuständig. Beide Zelltypen sind in der Wandstruktur vom Ösophagus bis zum Rektum sowie in Speicheldrüse, Pankreas und Gallenblase lokalisiert.

Das enterische Nervensystem liegt in der Darmwand in folgenden zwei Schichten:

- Plexus myentericus (Auerbach-Plexus), der zwischen der Ring- und Längsmuskulatur liegt und im Wesentlichen die Motorik steuert

- Plexus submucosis (Meissner-Plexus), der innerhalb der Tela submucosa liegt und für die Kontraktion der Zotten sowie die Sekretionsvorgänge von Galle und Bauchspeicheldrüse verantwortlich ist

\section{Nüchternmotilität: Cajal-Zellen triggern Entsorgung und Reinigung}

Bei der Steuerung der Motilität spielen die interstitiellen CajalZellen (ICCs), die auch als Schrittmacherzellen bezeichnet werden, eine wichtige Rolle. Sie produzieren durch die Aktivierung verschiedener Ionenkanäle und -pumpen einen basalen Organrhythmus (BER, basal electrical rhythm) - langsame, spontan aktive Potenzialwellen (slow waves) von 10-20 mV. Beim Menschen beträgt die Frequenz 3/min im Magen, 11-13/min im Jejunum und 6-8/min im terminalen Ileum. Diese Wellen allein induzieren noch keine Kontraktion der Darmmuskulatur. Sie erhöhen jedoch die Wahrscheinlichkeit, dass eintreffende Signale ein Aktionspotenzial auslösen. Dieser Mechanismus spielt insbesondere im Nüchternzustand (interdigestive Motilität) eine wichtige Rolle: Die motorischen Aktivitätswellen entsorgen zurückgebliebene
Nahrungsreste und reinigen somit den Darm. Sie laufen von oral nach aboral (vom Mund absteigend) und verhindern damit gleichzeitig die Wanderung von Bakterien aus dem Dick- in den Dünndarm. Unterstützt wird diese Reinigungsphase durch die Bildung von Magen-, Gallen- und Bauchspeicheldrüsensekret, die wie ein „Spülmittel“ wirken. Sobald wieder Nahrung aufgenommen wird, setzt die digestive Motilität ein, also der Transport und die Verdauung des Nahrungsbreis.

\section{Neuronale Steuerung: Vom sensorischen Input zum motorischen Output}

Die neuronale Steuerung des enterischen Nervensystems erfolgt über drei funktionelle Komponenten: den sensorischen Input, das verschaltete Interneuron und den motorischen Output. Sensorische Rezeptoren im Darm, zum Beispiel Mechano-, Nozi-, Chemooder Osmorezeptoren, nehmen Informationen über den aktuellen Funktionszustand des Darmes auf, zum Beispiel den Dehnungszustand der Darmwand nach einer Mahlzeit. Diese leiten sie über Interneurone, die den Hauptanteil der enterischen Nervenzellen ausmachen, an erregende oder hemmende Motoneurone weiter. Diese sind in der Lage, ein adäquates motorisches Reaktionsmuster auf den Reiz auszulösen, zum Beispiel Pendelbewegung, Segmentation oder Peristaltik. Exzitatorische Motoneurone leiten über den Transmitter Acetylcholin und die Substanz P die exzitatorischen Signale an den Muskel weiter und vermitteln eine Kontraktion. Inhibitorische Motoneurone vermitteln über Stickstoffmonoxid (NO), Purine (ATP) sowie vasoaktives intestinales Peptid (VIP) schließlich wieder eine Relaxation der glatten Muskulatur.

Serotonin (5-Hydroxytryptamin, 5-HT) ist einer der wichtigsten Neurotransmitter des enterischen Nervensystems und spielt eine zentrale Rolle für die gastrointestinale Motilität, Sekretion, und Sensibilität (Schmerz). Außerdem sorgt es dafür, dass der Appetit im Laufe einer Mahlzeit abnimmt. Im Darm wird es von Interneuronen und enterochromaffinen Zellen (Zellen zwischen den Enterozyten) der Darmschleimhaut (Mukosa) ausgeschüttet. Auslöser ist insbesondere eine mechanische, chemische oder elektrische Stimulation der Darmschleimhaut. Untersuchungen weisen darauf hin, dass Störungen im Serotoninmetabolismus wesentlich an der Pathogenese gastrointestinaler Erkrankungen beteiligt sind. Von den sieben bekannten Serotoninrezeptor-Familien mit ihren 21 Subtypen steht unter anderem der 5-HT3-Rezeptor im Fokus der neurogastroenterologischen Forschung. Die Gabe von 5-HT3-Rezeptor-Antagonisten wie Alosetron ist bei der Behandlung von funktionellen Darmerkrankungen daher sehr vielversprechend $[2,3]$.

\section{Wechselseitige Rückkopplung zwischen Gehirn und Darm}

Obwohl das enterische Nervensystem seine komplexen Reflexbögen (intestinal reflex circuits) eigenständig und ohne zentrale Steuerung aufbaut, kann es zusätzlich vom Parasympathikus (präganglionäre Fasern, Acetylcholin) und Sympathikus (postganglio- 
näre Fasern, Noradrenalin) moduliert werden. Dies gilt auch für afferente, sensorische Nervenfasern, welche die Informationen an das zentrale Nervensystem weiterleiten. Über diese Rückkopplungsschleifen hat das enterische Nervensystem entscheidenden Einfluss auf neuroendokrine, neuroimmunologische und neurosekretorische Interaktionen zwischen Gehirn und Darm.

\section{ENS-assoziierte Erkrankungen des Gastrointestinaltraktes}

Zu den Erkrankungen des enterischen Nervensystems gehören zum einen angeborene Defekte wie M. Hirschsprung, erworbene Schädigungen wie die Achalasie (Motilitätsstörung des Ösophagus), Infektionskrankheiten (Chagas-Krankheit) oder assoziierte Mitbeteiligungen im Rahmen von Systemerkrankungen wie Diabetes mellitus. In der Praxis kommen häufiger gastroenterologische Erkrankungen vor, darunter Divertikulose, Refluxerkrankung, Reizmagen (funktionelle Dyspepsie), Reizdarmsyndrom (irritables Colon), chronische Verstopfung und Stuhlinkontinenz. Diese Erkrankungen betreffen etwa 20-30\% aller Menschen.

\section{Morbus Hirschsprung: Megacolon durch parasympathische Dominanz}

Bei M. Hirschsprung - man spricht auch von Megacolon - handelt es sich um eine angeborene Erkrankung des enterischen Nervensystems des Dickdarms. Durch fehlende Ganglienzellen im Bereich des Meissner- und Auerbach-Plexus bilden sich vermehrt parasympathische Nervenfasern. Die verstärkte parasympathische Innervation fördert das Zusammenziehen des Ringmuskels im Bereich des Rektums. Es kommt häufig zu Obstipation und Darmverschluss. Die Krankheit manifestiert sich bereits nach der Geburt und kann lokal oder darüber hinaus zur Abwesenheit der neuronalen Ganglienzellen führen. Der proximale Darmanteil ist enorm erweitert. Symptome bei betroffenen Kindern sind eine beeinträchtigte Darmmotilität innerhalb der ersten beiden Monate, Bauchschmerzen, Verstopfung, Erbrechen, Blähungen und das Fehlen des ersten Stuhls des Neugeborenen innerhalb der ersten beiden Tage. Die Therapie der Wahl ist die operative Entfernung des betroffenen Darmabschnittes.

\section{Achalasie: Schluckvorgang durch Ösophagus-Nadelöhr gestört}

Die Achalasie ist eine primäre Motilitätsstörung der Speiseröhre. Sie äußert sich durch Schluckstörungen sowohl bei fester als auch bei flüssiger Nahrung. Im Extremfall kann die Nahrungsaufnahme so stark beeinträchtigt sein, dass es zum Flüssigkeitsmangel oder Gewichtsverlust kommt. Bei der Achalasie öffnet der untere Ösophagussphinkter nicht richtig. Aus bisher unklarer Genese degenerieren dort die inhibitorischen Ganglienzellen des Plexus myentericus. Therapeutisch kommen unter anderem medikamentöse Therapie, Ballondilatation der Speiseröhre, Botox-Injektionen oder die operative Erweiterung des Schließmuskels zur Anwendung.

\section{Chagas-Krankheit: Einzeller schädigt enterale Ganglienzellen}

Die Chagas-Krankheit ist eine Parasitose durch das Protozoon Trypanosoma cruzi und wird durch den Biss von Wanzen übertragen. Sie ist in vielen ländlichen Regionen Lateinamerikas endemisch und manifestiert sich unter anderem im Gastrointestinaltrakt. Durch die Infektion kommt es zu einer irreversiblen Schädigung von Ganglienzellen des Meissner- und Auerbach-Plexus. Folge sind nach anfänglichem Durchfall zunehmende Beeinträchtigungen der Darmmotorik bis hin zum Ileus. Die Chagas-Krankheit zählt zu den Reisekrankheiten. Sie wird medikamentös und meist stationär behandelt.

\section{Diabetes mellitus: \\ Neuropathie auch im Magen-Darm-Trakt}

Mit längerer Krankheitsdauer und schlecht eingestelltem Blutzucker tritt bei allen Diabetikern früher oder später eine Neuropathie auf, die jeden Nerv im Körper betreffen kann. So ist Diabetes mellitus ebenfalls mit einer Dysfunktion enterischer Schaltkreise assoziiert, die sich insbesondere in einer Gastroparese (Verlangsamung der Magenentleerung) niederschlägt. Der Pathomechanismus ist bisher unklar. Zahlreiche Studien konnten eine Reduktion der Nervenzellen des Meissner-Plexus im Rektumbereich nachweisen. Therapeutisch wird hier immer der Diabetes mellitus behandelt (siehe auch „Stoffwechsel in Schieflage“ in DHZ 1/2020, S. 48).

\section{Divertikulose: keine kausale Therapie}

Divertikel gehören zu den häufigsten Befunden im Gastrointestinaltrakt. Für die westlichen Industrienationen werden Prävalenzen um 10-15\% für Personen bis zum 50. Lebensjahr berichtet. Zwischen dem 50. und 70. Lebensjahr steigt der Wert auf etwa $30 \%$ und ab dem 70 . Lebensjahr auf rund $50 \%$. Patienten mit einer Divertikulose zeigen häufig eine sogenannte enterische Neuropathie mit einer erhöhten Anzahl von Gliazellen im Plexus myentericus und einer reduzierten Anzahl in beiden Nervenplexus. Man spricht von oligoneuronaler Hypoganglionose. Zu den Risikofaktoren zählen ein niedriger Ballaststoff- und hoher Fleischanteil in der Nahrung, Rauchen, Adipositas sowie die Behandlung mit NSAR, Steroiden und Opiaten. Auch genetische Faktoren haben eine erhebliche Bedeutung für die Ausbildung von Divertikeln. Die Therapie der Divertikulose erfolgt stadienabhängig. Grundsätzlich gibt es keine Therapiemöglichkeit zur Rückbildung der Divertikel. In meiner Praxis setze ich häufig stuhlregulierende Maßnahmen wie Flohsamenschalen, reichlich Flüssigkeitszufuhr (35 ml/ kg Körpergewicht), ausreichende Bewegung (3-5× 30 min Spazierengehen) und die Reduktion von rotem Fleisch und fetthaltiger Nahrung ein.

\section{Reizmagen und Reizdarm: Veränderter Funktionsmodus ohne Befund}

Als Reizmagen (funktionelle Dyspepsie) werden funktionell bedingte Störungen im Oberbauch bezeichnet, die keinem organisch bedingten, pathologischen Befund zugeordnet werden können. Sie 
werden meist als nervöse Magenbeschwerden (Gastropathia nervosa) wahrgenommen. Konventionell kommen Protonenpumpenhemmer, Antibiotika (bei Helicobacter-pylori-Infektion), Antidepressiva und Psychotherapie zur Anwendung. Beim Reizmagen wird dem Neurotransmitter Serotonin eine Schlüsselrolle zugesprochen. Zwei neue Arzneimittel zur Behandlung des Reizdarmsyndroms greifen daher an Serotoninrezeptoren an: der selektive 5-HT3-Rezeptorantagonist Alosetron und der partielle 5-HT4Agonist Tegaserod.

Die Ausschlussdiagnose Reizdarmsyndrom (RDS) wird dann gestellt, wenn die auftretenden Verdauungsbeschwerden - darunter Diarrhö und/oder Obstipation - die Lebensqualität maßgeblich beeinträchtigen und zum Zeitpunkt der Diagnosestellung mindestens drei Monate bekannt sind. Diagnostische Methoden beinhalten eine Sonografie des Abdomens, eine Gastro- sowie Koloskopie und einen $\mathrm{H} 2$-Atemtest.

Mit dem Reizdarmsyndrom ist eine gestörte Darmmotilität verbunden. Dabei können eine erhöhte Eigenbewegung (Diarrhö dominanter Typ, RDS-D) oder verlangsamte Kontraktionen (Obstipation dominanter Typ, RDS-O) dominieren.

Auch eine veränderte Schleimhautsekretion und -durchlässigkeit wird als Ursache des Reizdarmsyndroms diskutiert. Heute ist bekannt, dass bei etwa 20\% der Patienten Infektionen des MagenDarm-Traktes vorausgegangen sind (postdysenterisches Reizdarmsyndrom). Die Aktivierung von immunologischen Kaskaden und den daraus entstehenden lokalen Entzündungen kann eine veränderte Barrierefunktion und erhöhte Permeabilität selbst Monate nach dem akuten Infekt-Trigger bewirken.

\section{Therapie des Reizdarmsyndroms: Reaktionen wieder regulieren}

Die Therapie richtet sich immer nach der klinischen Symptomatik und dem Leidensdruck des Patienten. Kombinationen mehrerer Therapiemöglichkeiten sind häufig sinnvoll. Im Folgenden soll das Reizdarmsyndrom als Leiterkrankung bei Störungen im enterischen Nervensystem im Fokus stehen.

\section{Orthomolekulare Therapie: Vitamin D und Omega-3-Fettsäuren}

Aus der Orthomolekularen Medizin ist bekannt, dass Vitamin D die Umwandlung der essenziellen Aminosäure Tryptophan zu Serotonin reguliert. Dieses spielt wiederum eine Schlüsselrolle für die Darmmotorik und -sekretion. Daher sollten Reizdarmpatienten auf ihre Vitamin-D3-Versorgung achten.

Auch Omega-3-Fettsäuren beeinflussen den Serotoninmetabolismus. Patrick und Ames [4] konnten zeigen, dass Eicosapentaensäure (EPA) die Serotoninproduktion durch die Reduktion hemmender E2-Prostaglandine erhöht. Docosahexaensäure (DHA) stimuliert ihrerseits diverse Serotoninrezeptoren. Bei Reizdarmpatienten wird daher empfohlen, täglich 2000-3000 mg Omega3-Fettsäuren (EPA und DHA) einzunehmen. Ideal ist ein Präparat mit einem hohen Anteil der stärker entzündungshemmenden EPA (zum Beispiel der Fa. Norsan).

\section{Scharfes Capsaicin: \\ Desensibilisierung der Darmschleimhaut}

Viele Reizdarmpatienten weisen eine erhöhte Sensitivität gegenüber abdominalen Schmerzen auf. Schleimhautbiopsien ergaben eine erhöhte Anzahl an Capsaicinrezeptoren. Capsaicin ist für die Schärfe von Chili verantwortlich. Therapeutisch gibt es erste Versuche, in denen es als Arzneimittel zur Diagnose einer bei Reizdarm auftretenden Hypersensibilität und anschließenden Desensibilisierung eingesetzt wird. Untersuchungen ergaben für eine Dosis von 2,5 g Chilipulver pro Tag, verteilt auf drei Gaben je 15 min vor den Mahlzeiten, positive Ergebnisse [5]. Biologischer Hintergrund ist, dass Capsaicin selektiv die Aktivität von afferenten nozizeptiven C-Fasern reduziert. Bei meinen Patienten verabreiche ich im Laufe von sechs Wochen entsprechend ihrer persönlichen Toleranz die tägliche Dosis von beginnend 0,5 bis 2,5 g täglich. Die individuelle Menge wird dann über einen längeren Zeitraum gehalten, bis die Patienten schmerzfrei beziehungsweise deutlich schmerzreduziert sind.

Phytotherapeutisch eignen sich ergänzend zum Beispiel Iberogast $^{\circledR}$ (Fa. Bayer, $3 \times 20$ Tr.) und Carmenthin ${ }^{\circledR}$ (Fa. Dr. Willmar Schwabe, $2 \times 1$ Kps.), eine Wirkstoffkombination aus Pfefferminzund Kümmelöl.

\section{Hemmung der HPA-Stress-Achse entlastet den Darm}

Die Hypothalamus-Hypophysen-Nebennierenrinden-Achse (HPAAchse, für Hypothalamic-Pituitary-Adrenal Axis) nimmt eine wichtige Stellung in der multifaktoriellen Pathogenese des Reizdarmsyndroms ein. Insbesondere psychischer Stress bedeutet eine anhaltende Ausschüttung des Cortico-Releasing-Hormons CRH im Hypothalamus als erster Stufe. Wird sie durch die Gabe eines CRH-Antagonisten (bislang nur experimentell) blockiert, kommt es zu einer deutlichen Verbesserung von Darmmotilität, Angst und Schmerzen [6].

\section{Unsichtbares sichtbar machen - die Herzratenvariabilität}

Um den Stress sichtbar zu machen, messe ich bei meinen Patienten die Herzratenvariabilität (HRV), also den natürlichen Unterschied der Zeitspanne zwischen aufeinanderfolgenden Herzschlägen. Bei chronischem Stress arbeiten Parasympathikus und Sympathikus nicht mehr antagonistisch, und die Herzratenvariabilität ist erniedrigt. Bei einer pathologischen Funktionslage bleibt sie selbst in einer anschließenden Ruhesituation auf dem niedrigen Wert [7].

Zur Regulation der HPA-Achse setze ich in meiner Praxis das Höhenluft-Zelltraining (IHHT, Intermittierende-Hypoxie-Hyperoxie-Therapie) ein. Die IHHT führt nicht nur zu einer Erhöhung der Zellenergie durch Stabilisierung der Mitochondrien, sondern sie verbessert auch die Stressresistenz und Regenerationsfähigkeit des Organismus.

\section{FODMAP-reduzierte Diät: Noxen aus dem Dickdarm verbannen}

Das Akronym FODMAP steht für Fermentable Oligo-, Di-, Monosaccharides And Polyols und beschreibt bestimmte Zucker- und 


\section{ÜBERSICHT}

\section{FODMAP-arme Nahrungsmittel}

Eine Übersicht FODMAP-armer Nahrungsmittel [1] gibt Patienten eine wichtige Orientierung an die Hand. Folgende Vertreter (Auswahl) weisen besonders geringe Gehalte auf.

\section{Früchte}

Ananas, Bananen, Clementinen, Erdbeeren, Grapefruit, Heidelbeeren, Himbeeren, Honigmelonen, Kiwis, Limetten, Mandarinen, Netzmelonen, Orangen, Weintrauben, Zitronen

\section{Gemüse}

Auberginen, Brokkoli, Chili, Chinakohl, Erbsen, Fenchel, Grüne Bohnen, Gurken, Ingwer, Karotten, Kartoffeln, Kichererbsen, Kohlrabi, Kopfsalat, Kürbis, Mais, Mangold, Okra, Oliven, Paprika, Pastinaken Petersilie, Rosenkohl, Sellerie, Spinat, Süßkartoffeln, Tomaten, Weißkohl, Zucchini

\section{Getreide, Samen, Nüsse}

Buchweizen, Dinkel, Flohsamen, glutenfreie Getreideprodukte (zum Beispiel mit Kartoffel-, Reis- oder Maismehl), Hafer, Haselnüsse, Hirse, Kürbiskerne, Mandeln, Quinoa, Reis, Sesam, Sonnenblumenkerne, Tapioka, Walnüsse

\section{Milchprodukte}

Brie, Butter, Camembert, Feta, Hartkäse, Kokosmilch, laktosefreie Milch(-Produkte), Mozzarella, Ricotta, Sojamilch

\section{Öle}

Sojaöl, Rapsöl, Olivenöl, Erdnussöl, Reiskleieöl, Sesamöl

\section{Andere Nahrungsmittel}

Bier, Eier, Essig, Fisch, Gelatine, Gemüsebrühe, Hühnchen, Kakao, Kokosnuss, Lamm, Meeresfrüchte, Rindfleisch, Schokolade (dunkel), Senf, Sojasauce, Tofu, Truthahn, Wein (trocken)

Alkoholverbindungen wie Fruktose, Laktose, Sorbitol und Xylitol in Lebensmitteln. Bei der FODMAP-reduzierten Diät werden Lebensmittel weggelassen, die durch einen hohen FODMAP-Gehalt häufig Verdauungsprobleme verursachen und durch FODMAP-arme Alternativen ersetzt (siehe Übersicht). Diese vorübergehende Ernährungsanpassung basiert auf einem neuen Diätprinzip, das zur Vermeidung und Behandlung von Verdauungsbeschwerden entwickelt wurde. Denn diese Gruppe kurzkettiger Zuckerarten gelangt unverdaut in den Dickdarm, da der Dünndarm diese nur unzureichend verdauen kann. Dort werden sie von Bakterien fermentiert und produzieren Stoffwechselendprodukte wie Methylglyoxal und Gase, die bei einem Reizdarm zusätzliche Beschwerden verursachen. Durch die Reduzierung FODMAP-reicher Nahrungsmittel vermindern sich belastende Reize und damit die Verdauungsbeschwerden. Lebensmittel, gegen die eine Unverträglichkeit oder Allergie besteht, dürfen nicht in die FODMAP-Diät aufgenommen werden.

Bei etwa $80 \%$ meiner Patienten kommt es zwei bis vier Wochen nach der Ernährungsumstellung zu einer deutlichen Symp- tombesserung und einem Anstieg der Lebensqualität. Sie bekommen zudem ein Instrument an die Hand, mit dem sie ohne fremde Hilfe und zusätzlichen finanziellen Aufwand ihre Situation maßgeblich gestalten können.

\section{Fazit: Verlernte Reaktionen wieder erlernen}

Auch wenn die Pathomechanismen im Detail noch nicht hinreichend erforscht sind, so hat das enterische Nervensystem bei chronischen gastrointestinalen Beschwerden verlernt, richtig auf sensorische Reize zu reagieren. Eine Therapie erfordert immer ein individuelles Konzept. Hierbei zählt eine Kombination aus Ernährungsumstellung, Stressreduktion und guter orthomolekularer Versorgung zu den aussichtsreichsten Ansätzen.

므 Dieser Artikel ist online zu finden: http://dx.doi.org/10.1055/a-1192-2650

\section{目 Literatur}

[1] Storr M. Der Ernährungsratgeber zur FODMAP-Diät. München: Zuckschwerdt; 2015

[2] Gershon MD. Review article: serotonin receptors and transporters - roles in normal and abnormal gastrointestinal motility. Aliment Pharmacol Ther 2004

[3] Sikander A, Rana SV, Prasad KK. Role of serotonin in gastrointestinal motility and irritable bowel syndrome. Clin Chim Acta 2009

[4] Patrick RP, Ames BN. Vitamin D and the omega-3 fatty acids control serotonin synthesis and action, part 2 Relevance for ADHD, bipolar disorder, schizophrenia, and impulsive behavior. FASEB J. 2015

[5] Bortolotti M, Coccia G, Grossi G, Miglioli M. The treatment of functional dyspepsia with red pepper. New England Journal of Medicine 2002

[6] Sargami et al. Effect of a corticotropin releasing hormone receptor antagonist on colonic sensory and motor function in patients with irritable bowel syndrome. Gut 2004; 53

[7] Nixdorf U. Check-Up-Medizin: Prävention von Krankheiten - Evidenzbasierte Empfehlungen für die Praxis. Stuttgart: Thieme; 2009

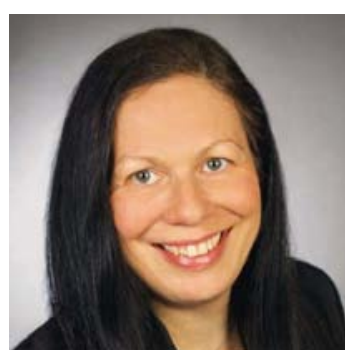

HP DR. RER. NAT. KEREN GRAFEN

Keren Grafen ist Neurobiologin und Heilpraktikerin. Biologiestudium, Forschungsstudium und Promotion an der Universität Bielefeld, Lehrstuhl für Neuroanatomie und Lehrstuhl für kognitive Neurowissenschaften. Seit 2013 arbeitet sie selbstständig in eigener Praxis. Wissenschaftliche Themenfelder: Einfluss frühkindlicher Erfahrungen auf die Hirnreifung, Auswirkungen von Drogen und Stress auf emotional und kognitiv verarbeitende Hirnstrukturen. Sie ist Dozentin für Neurologie und Sinnesphysiologie sowie Autorin zahlreicher wissenschaftlicher Publikationen.

E-Mail: info@praxis-biomed.de 